\title{
Belphégor
}

\section{Genèse et circulations d'un genre populaire en régime médiatique : le cas du Country Noir}

Alice Jacquelin

\section{(2) OpenEdition}

Journals

Édition électronique

URL : https://journals.openedition.org/belphegor/3803

DOI : 10.4000/belphegor.3803

ISSN : 1499-7185

Éditeur

LPCM

Référence électronique

Alice Jacquelin, « Genèse et circulations d'un genre populaire en régime médiatique :

le cas du Country Noir », Belphégor [En ligne], 19-1 | 2021, mis en ligne le 28 juin 2021, consulté le 07

juillet 2021. URL : http://journals.openedition.org/belphegor/3803 ; DOI : https://doi.org/10.4000/

belphegor.3803

Ce document a été généré automatiquement le 7 juillet 2021.

\section{(c) ()) (9)}

Belphégor est mis à disposition selon les termes de la Licence Creative Commons Attribution - Pas d'Utilisation Commerciale - Pas de Modification 4.0 International. 


\title{
Genèse et circulations d'un genre populaire en régime médiatique : le cas du Country Noir
}

\author{
Alice Jacquelin
}

1 L'expression "Country Noir» est née en 1996 sous la plume de Daniel Woodrell, un auteur étasunien de romans noirs, qui a sous-titré son roman Give Us a Kiss, « A Country Noir $»^{1}$. Give Us a Kiss est le premier des cinq romans des Monts Ozarks (1996-2013), qui ont tous pour toile de fond ces montagnes de moyenne altitude situées à la frontière des états du Kansas, du Missouri, de l'Oklahoma et de l'Arkansas. La série romanesque des Monts Ozarks fonctionne par opposition à la «Trilogie du Bayou» de Woodrell, écrite antérieurement (1986-1992), et qui se déroule en Louisiane dans le Sud des ÉtatsUnis. Give Us a Kiss a été publié en France dans la collection Rivages/Noir en 1998 avec pour titre traduit Faites-nous la bise, et pour sous-titre "un roman noir rural». La registre du Country Noir est donné grâce aux premières pages programmatiques de Give Us a Kiss, ici dans la traduction de Michèle Valencia :

Au bout d'environ cent soixante kilomètres, je bifurquai vers l'est et pénétrai dans la région des Ozarks, qui est exactement le contraire d'un complexe urbain. Tout y est : prairies et montagnes, arbres et terre rouge rocailleuse. À voir les maisons, on se dit qu'elles ont été construites par différentes générations aux notions d'architecture variées qui se mêlent toutefois pour donner un unique bâtiment fantasque dont les ailes semblent presque avoir été ajoutées pour contredire les précédents bâtisseurs. On commence à voir les poules dans les cours, d'immenses jardins, des fauteuils à bascule dans les vérandas et divers véhicules qui ont rouillé en se fondant tellement bien dans le paysage qu'ils ont pris un petit air indigène. Un certain nombre de cabanes, malmenées par les intempéries, penchent dangereusement mais ont toujours leur utilité ces jours redoutables où la fosse septique refoule ${ }^{2}$.

2 Le narrateur souligne le caractère profondément rural de la région des Ozarks qu'il traverse, sans jamais s'abimer dans la niaise contemplation du panorama. Il se moque d'ailleurs des clichés "paysagers" par l'expression englobante "tout y est » et l'énumération paratactique expéditive qui s'ensuit. Il donne ensuite une vision 
humaine et sociale de l'espace en décrivant les habitations pauvres et quelques éléments de détail qui construisent l'espace du Country Noir en quelques coups de pinceaux : les poules de la basse-cour, les rocking-chairs traditionnels du Sud et les épaves de carrosserie stéréotypiques de l'imagerie sudiste. Le narrateur achève de miner l'évocation, qui aurait pu être bucolique, en évoquant une réalité scatologique qui, alliée au déictique «ces jours redoutables » et au présent de vérité général « où la fosse septique refoule", fonctionne comme une expérience partagée par toute la population des Ozarks. Le Country Noir n'a rien de champêtre, la nature y est rude comme les habitants et les histoires qui s'y déroulent.

Daniel Woodrell pose ici les bases littéraires du Country Noir et des marges rurales qu'il explore. Cependant, quelques années plus tard et face à l'immense succès de l'étiquette "Country Noir », Daniel Woodrell va renier cette appellation qu'il a pourtant contribué à créer ${ }^{3}$. Comment se construit la catégorie de Country Noir dans le champ médiatique et ce, malgré les réticences de son inventeur, Daniel Woodrell? Comment $l^{\prime}$ « interdiscours médiatique ${ }^{4}$ » participe-t-il de la fortune d'une appellation ? Comment un sous-genre mineur du roman noir - et les auteur-tricess qui l'incarnent - gagnent-ils en légitimité dans le champ culturel ? Comment la circulation des œuvres, notamment entre la France et les États-Unis, ainsi que l'extension de l'étiquette à d'autres supports de la culture populaire comme le cinéma et les séries télévisées, participent-elles de la consolidation d'un paradigme encore mouvant? Comment penser de manière dynamique et plastique la naissance et la formation d'un genre populaire transnational et intermédial?

Notre objectif est ici d'envisager le genre et la généricité à la manière de Matthieu Letourneux à la fois comme une "pratique historique", définie par ses usages, et comme un produit médiatique ${ }^{5}$. L'approche médiatique du genre s'articule à différentes échelle et nous permet d'abord de circonscrire un "champ" occupé discursivement dans la critique du Country Noir au sein de l' « interdiscours médiatique ", elle permet aussi de définir un "régime médiatique " plus large, lié à la visibilité et la peopolisation des auteur-trice's de Country Noir obligée's d'adopter une image médiatique, et enfin la " transmédiagénie ${ }^{6}$ » des récits de Country Noir leur permet de se décliner sur divers supports médiatiques de la culture populaire. Il s'agira non plus de considérer la généricité artistique dans une perspective formaliste et systématique mais plutôt dans une perspective fluide et dynamique. Les contours du Country Noir sont encore flous : la terminologie reste instable, le corpus romanesque et transmédiatique est mouvant, les discours ne s'accordent pas. Le Country Noir en est encore au stade de la négociation entre les différente's acteur.trice's du champ, des producteur.trice's aux récepteur.trice's en passant par les diffuseur-euse-s.

Nous allons voir comment le genre du Country Noir se construit, médiatiquement, selon ces trois modalités. Il émerge tout d'abord à travers un interdiscours médiatique en France et aux États-Unis, ce qui traduit une négociation transnationale entre les différente-s acteur.trice's du champ du Noir. Le Country Noir devient ensuite un produit qui s'inscrit dans le régime médiatique spécifique des stratégies de communication des maisons d'édition, impliquant la mise en place de "postures ${ }^{7}$ " d'auteur.trice's. Enfin, le Country Noir se consolide comme un genre intermédial qui circule de façon transnationale sur différents supports médiatiques typiques de la culture populaire contemporaine : le roman policier, le cinéma et les séries télévisées. 


\section{Stabilisation progressive du « Country Noir » dans l'interdiscours médiatique}

6 Si elle naît sous la plume de Daniel Woodrell, l'expression "Country Noir " est cependant reprise dans l'interdiscours médiatique qui englobe tou'te's les acteur.trice-s du champ culturel. Ces discours populaires sont intéressants pour le-la chercheureuse parce qu'ils fournissent un excellent indicateur des étiquettes génériques en formation, de leur réception, et de leurs utilisations par les lecteur.trice's et récepteur.trice's. Nous allons, dans un premier moment voir comment la catégorie de «Country Noir » est reprise dans le discours amateur étasunien sur les blogs, les sites internet et les sites de recommandation de films (IMDB) et de romans (Goodreads) pour ensuite analyser, grâce à un moissonnage d'archives à la Bilipo ${ }^{8}$, comment la critique professionnelle, les maisons d'édition et les libraires s'en emparent en France.

7 D'un côté comme de l'autre de l'Atlantique, les maisons d'édition, les critiques littéraires, les amateur.trice's, les fans, ainsi que certain'e's auteur.trice's eux'ellesmêmes s'emparent de cette étiquette de "Country Noir " pour qualifier toute une production de romans, de séries télévisées et de films noirs contemporains. On trouve dans ce vaste ensemble des œuvres comme les nouvelles de Frank Bill (Crimes in Southern Indiana, 2011), les romans de Chris Offutt (Country Dark, 2018), de Benjamin Whitmer (Cry Father, 2016), d'Andrea Portes (Hick, 2013) ou de Larry Brown (Joe, 2003), mais aussi des films des frères Cohen (No Country for Old Men, 2007) et de Jeff Nichols (Mud, 2012), ou encore des séries télévisées comme Breaking Bad (2008-2013), True Detective (2014-) ou Ozark (2017-). Give Us a Kiss n'est donc pas le seul représentant du Country Noir même s'il en est très certainement l'une des œuvres séminales. Le Country Noir est accolé, notamment par le discours étasunien amateur, à de nombreuses autres œuvres sur tous les supports médiatiques. On retrouve sur Internet des blogs, des posts et des sites qui référencent les œuvres appartenant alternativement au Southern Gothic, au Country Noir, ou au Rural Noir, ces œuvres étant bien souvent les mêmes. Dans le discours amateur, on observe toute une nébuleuse d'étiquettes génériques, de registres, de références communes et de mentions géographiques qui encerclent le Country Noir. Pour ne donner qu'un exemple de ce foisonnement d'appellations qui gravitent autour du Country Noir, voici la description d'une liste de recommandation de films du contributeur Schwenkstar intitulée «Rural-country thrillers » sur le célèbre site IMDB (Internet Movie Database) :

L'idée de cette liste: du Southern Gothic, des films d'horreur dans l'arrière-pays, des films noirs hillbilly, des mystères de petites bourgades, un parfum de ruralité - des histoires criminelles typiques au milieu de gars locaux. Certains films ressemblent à des néo-westerns, ou à des country noirs, et d'autres à des thrillers survivalistes?

Cette prolifération onomastique est l'un des signes d'un genre artistique en constitution. Dans Autopsies du roman policier, Uri Eisenszweig explique par exemple que l'étiquette générique de "roman policier » fut très longue à se stabiliser, concurrencée qu'elle était à l'époque par d'autres catégories comme la "criminal romance » et la «detective story» en Angleterre ou encore le « roman judiciaire » en France ${ }^{10}$. Matthieu Letourneux remarque la même " hétérogénéité terminologique ${ }^{11}$ " à propos des récits de science-fiction au début du $\mathrm{Xx}^{\mathrm{e}}$ siècle $^{12}$. Le « Country Noir » est donc encore un genre en émergence et en mutation. On trouve de nombreuses autres expressions qui recoupent celle de "Country Noir», notamment sur le site Goodreads de 
recommandations littéraires : les listes de romans appartenant au Rural Noir (le Noir rural), à la Hick Lit (littérature de péquenaud), à la Redneck Lit (littérature de redneck) et à la Grit Lit (littérature du Sud) reprennent en fait bien souvent les mêmes auteur.trice's que la liste de romans Country Noir. Certains auteurs comme Donald Ray Pollock ou Harry Crews relèveraient de la branche que les critiques identifient comme la littérature Redneck, d'autres comme Ron Rash, James Crumley ou James Lee Burke seraient plus proches du Nature Writing, d'autres encore comme Daniel Woodrell ou Chris Offutt seraient affiliés au Southern Gothic.

III. 1: Benoît Minville, Rural Noir, Paris, « Série Noire », Gallimard, 2016

III. 2: Chris Offutt, Country Dark, New-York, Grove Press, 2018
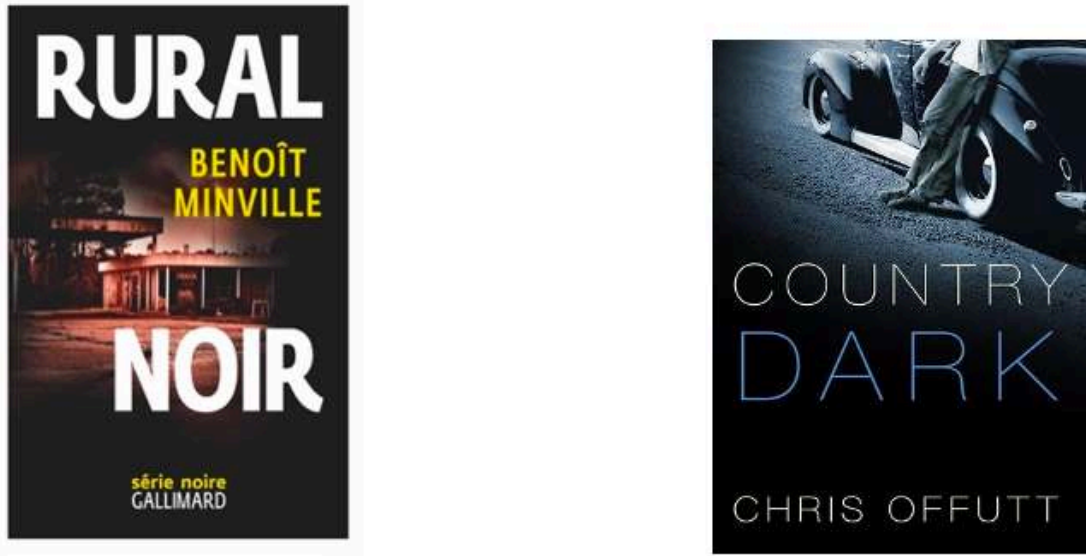

$10 \mathrm{Au}$ sein de cette nébuleuse d'appellations, les étiquettes de "Country Noir » et de « Rural Noir » semblent non seulement les plus récurrentes dans le discours médiatique mais aussi les plus englobantes - puisqu'il s'agit d'expressions qui qualifient aussi bien des productions textuelles que visuelles - et elles sont surtout les plus stables puisqu'elles transitent volontiers entre la France et les États-Unis. En 2016, le premier roman noir du Français Benoît Minville, situé dans la Nièvre et publié à la Série Noire, est intitulé Rural Noir. Quatre autres romans noirs sortent en France en 2016, qui s'inscrivent dans cette veine du Rural Noir et qui sont d'ailleurs parfois référencés comme tels par les critiques littéraires : D'Ombres et de flammes de Pierric Guittaut (coll. Série Noire) remonte une filière mafieuse en Sologne, Aux animaux la guerre (coll. Babel Noir), premier roman de Nicolas Mathieu - auteur qui gagnera l'année suivante le prix Goncourt pour son second roman Leurs enfants après eux - dépeint la fermeture d'une usine dans les Vosges, Battues d'Antonin Varenne suit un garde-chasse, Rémi Parrot, au cœur d'une bataille immobilière dans une région reculée et enfin Grossir le ciel de Franck Bouysse - que les critiques présentent souvent dans le père du roman noir rural français - raconte un drame fratricide et paysan dans les Cévennes. Le Country Noir se situe donc encore au sein d'une nébuleuse terminologique et au croisement de plusieurs lignes de forces qui définissent un "champ » littéraire et culturel, au sens 
bourdieusien du terme. Les négociations discursives à l'œuvre sont en fait autant de manières pour le Country Noir de se trouver une place dans le champ.

11 À la suite de cette observation des blogs, sites critiques et sites de recommandation littéraires et cinématographiques étasuniens, nous nous sommes penchée sur la réception française grâce à une autre méthode : celle du moissonnage de plus de quatre cents archives de presse française collectées à la BiLiPo (Bibliothèque de Littératures Policières ${ }^{13}$ ) et à leur traitement par le biais d'outils numériques ${ }^{14}$. Ces deux méthodes le moissonnage Internet étasunien et celui des archives françaises - visent à décrire les modalités d'émergence d'un genre populaire et sa progressive conquête d'une légitimité jusqu'au succès populaire, éditorial et médiatique qu'il connaît aujourd'hui. Dans ces archives, nous avons pu observer de plus près les différents discours des acteur.trice's du champ culturel français. L'objectif est d'analyser la fortune ou l'infortune de certaines expressions en fonction de l'instance discursive émettrice, qu'il s'agisse de libraires, de critiques, de communiqués de presse des maisons d'édition ou de paroles d'auteur.trice's dans des entretiens. Nous avons entrepris cette recherche sur sept lexèmes radicaux: «nature », "profond*", "paysage*", «rura*», « compagn*», « écolo* » et « country ».

\section{3: Analyse de mots-clés en fonction par instances discursives grâce à l'outil « Tendancy »}

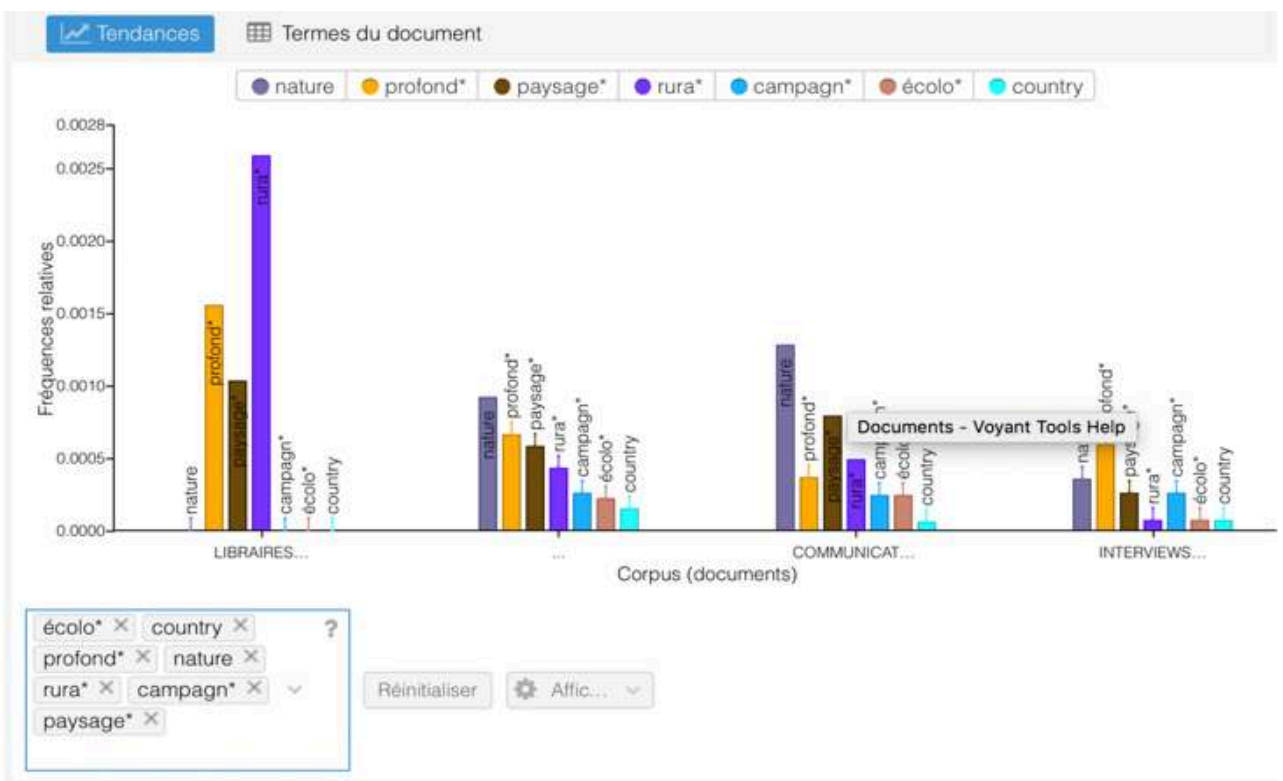

On peut voir, grâce à ce graphique en colonnes, la proportion d'utilisation de chaque lexème radical au sein de chaque discours : libraires, critiques (journalistes littéraires), communications (maisons d'édition) et interviews (auteurtrice's) (le second corpus dans l'ordre de lecture est en fait le corpus de critiques littéraires). Quatre éléments signifiants ressortent de cette analyse quantitative. Premièrement, le corpus «Libraires » étant particulièrement réduit, on note l'absence de quatre des lexèmes de notre étude, les sept lexèmes étant présents dans les trois autres corpus. Deuxièmement, le terme " country » et le lexème " écolo* » sont proportionnellement les moins représentés dans l'interdiscours global, par rapport au lexème "profond*" par exemple. Ce constat s'explique d'une part par le fait que les deux premiers concepts sont relativement récents, et d'autre part parce que les termes associés à " profond* " permettent de qualifier un décor rural de façon sociologique et littéraire: on parle 
aisément de "France profonde" ou d'"Amérique profonde", notamment chez les critiques et les libraires. L'adjectif connote en outre la marginalité, éventuellement sociale autant que géographique, ainsi qu'une certaine mise à distance péjorative, que les auteurs s'attachent à décrire par rapport au centre fantasmé que sont les villes. Troisièmement, le terme de « nature » est très présent au sein des discours des maisons d'édition et des journalistes, ce qui est là encore significatif puisque ces deux instances peuvent traiter en commun d'un concept comme le Nature Writing, catégorie sans doute trop académique pour des auteur.trice's ou des libraires. Quant au lexème "campagn*", il est représenté dans presque tous les discours (sauf chez les libraires) de façon réduite mais stable, en moindre proportion cependant que le terme "paysage ", sans doute plus évocateur. Quatrièmement, c'est le lexème "rura* " qui connaît la destinée la plus étonnante: très présent chez les libraires, il est moyennement utilisé par les communicant·es des maisons d'édition et les organes de presse, et presque inexistant chez les auteur.trice-s. Le terme est très géographique, ancré dans le réalisme et donc peu vendeur, sans doute moins utilisable par la communication, les critiques littéraires et les créateur.trice's.

$\mathrm{Au}$ sein de cet interdiscours, le discours des maisons d'édition à travers les communiqués de presse, nous intéresse tout particulièrement. Lorsque l'on quitte le distant reading quantitatif pour se pencher sur le close reading qualitatif ${ }^{15}$, on relève deux acceptions particulièrement intéressantes de l'expression "Country Noir", qui se réfèrent à des romans français et non étasuniens. La première est associée au roman de Benoît Minville Rural Noir publié à la Série Noire en 2016 : le roman est accolé au Country Noir à la fois dans le communiqué de presse du grand format mais aussi dans celui du format poche. Le communiqué de presse de l'édition grand format dit ceci : " Dans la tradition du "country noir américain", où territoires ruraux et laissés-pourcompte côtoient ceux dont on parle peu au milieu d'une nature "préservée" ou en friche, Rural Noir est aussi un hommage à Joe R. Lansdale, Larry Brown ou Ron Rash ${ }^{16}$ ». Quant au communiqué de presse en format proche, il mentionne « un récit porté par les goûts de ce nouvel auteur, entre littérature américaine emprunté du style country noir et les références à la culture heavy metal ${ }^{17}$ ". La seconde acception est accolée au roman d'Hervé Le Corre Prendre les loups pour des chiens, édité chez Rivages/Noir en 2017 : "Entre le "country noir" des Américains et le roman de terroir à la française, Le Corre fait entendre sa voix inimitable », dit le communiqué de presse. Les grandes collections historiques, la Série Noire et Rivages/Noir réutilisent donc cette étiquette de "Country Noir " dans le cadre de leur plan marketing comme un argument de vente des polars français aussi bien d'un jeune auteur comme Minville que d'un auteur confirmé comme Le Corre. Pour les romans français, la référence aux romans noirs étasuniens a toujours été un outil de légitimation mais il est intéressant de noter que cette relative nouvelle catégorie de Country Noir est assise ici comme une référence d'autorité alors qu'elle est encore au stade de nébuleuse dans le discours amateur étasunien, comme nous l'avons constaté plus haut.

\section{Postures et stratégies de communication en régime médiatique}

En France, certaines collections de moins grande ampleur et certaines maisons d'éditions françaises moins historiques se construisent quant à elles entièrement sur le 
concept du Country Noir. Oliver Gallmeister a fondé en 2006 la maison d'édition du même nom au sein de laquelle il publie de grands textes américains aussi bien classiques que contemporains. Initialement, la maison s'est fondée sur deux collections : la collection "Nature Writing» et la collection « Néo-Noire». Ces deux collections donnaient le ton à la maison d'édition en alliant une certaine vision du Noir étasunien à une localisation non-urbaine, qu'il s'agisse de grands espaces ou de territoires ruraux. Ce regard d'oliver Gallmeister, à la fois tourné vers le polar criminel et la Nature au sens large, confère au catalogue de la maison d'édition une coloration Country Noir. Gallmeister joue ainsi le rôle de passeur des textes étasunien de Country Noir comme l'avait été avant lui Christian Bourgois pour le Nature Writing et Marcel Duhamel pour le roman noir en général ${ }^{18}$ au sortir de la Seconde Guerre mondiale. On retrouve la même alliance entre polar et ancrage rural, mais avec des textes écrits par des Français.e.s cette fois-ci, chez un éditeur comme Cyril Herry qui a monté la collection "Territori » au sein de la maison d'édition Ecorce, en partenariat avec La Manufacture de livres. Cette collection avait pour objectif initial d'éditer des polars situés dans la région limousine ${ }^{19}$. La collection a connu notamment le succès avec les romans de Franck Bouysse puis avec Battues d'Antonin Varenne (2015). Cyril Herry s'est toujours revendiqué du Nature Writing étasunien sans toutefois avoir la volonté de le transposer en France: c'est pourquoi l'expression Country Noir lui paraît plus cohérente pour décrire ces polars français ruraux ${ }^{20}$.

Si la catégorie du Country Noir se comprend donc comme une co-construction francoétasunienne entre les discours amateur étasunien d'un côté et la communication des maisons d'édition françaises de l'autre, un autre élément de l'interdiscours médiatique nous paraît intéressant à observer : il s'agit des postures assignées aux auteur.trice's de Country Noir. Marie-Laure Rossi explique que les auteur.trice's contemporaine's se situent désormais au sein d'un "régime médiatique ", c'est-à-dire dans une ère de la " communication de masse » qui « concerne l'information journalistique, la promotion marchande, certes, mais aussi toutes les productions dites "culturelles", devenues déterminantes pour la structuration de l'imaginaire collectif contemporain ${ }^{21}$ ". Cependant, Rossi n'analyse pas la notion de "posture " dans le champ culturel et littéraire, or ces mises en scène biographiques et médiatiques sont très instructives. Jérôme Meizoz définit les "postures littéraires ${ }^{22}$ » comme une "manière singulière d'occuper une position objective dans un champ, balisée quant à elle par des variables sociologiques ${ }^{23}$ ». Il s'agit donc d'une présentation de soi située dans des conditions sociales d'énonciation spécifiques, en l'occurrence le « régime médiatique ».

L'analyse des trajectoires des auteurtrice's de Country Noir et les processus de légitimation présents dans les communiqués de presse à notre disposition sont très signifiants. De manière générale, les éléments biographiques valorisent des écrivain·e·s «durees-à-cuire» selon plusieurs modalités de légitimation. La première de ces modalités est l'origine sociale modeste ou ouvrière des auteur.trice's, comme on le voit très bien pour le cas de Donald Ray Pollock dans la communication d'Albin Michel pour Le Diable tout le temps:

Donald Ray Pollock est né en 1954 dans le sud de l'Ohio. Il a grandi dans une petite ville du nom de Knockemstiff. Il a abandonné les études à l'âge de dix-sept ans pour travailler dans un abattoir, avant d'être employé pendant trente-deux ans dans une fabrique de pâte à papier à Chillicothe, Ohio, un de ces métiers que l'on se transmet de père en fils au nom du syndicalisme ${ }^{24}$. 
17 La mention de la filiation s'inscrit dans une généalogie ouvrière qui fonctionne là comme une forme de légitimation. On note souvent un rejet des stratégies de légitimation de la littérature majeure, qui fonctionne avec un système de prestige fondé sur le statut symbolique. Ici à l'inverse, c'est l'appartenance aux marginalités au cœur des romans Country Noir - qui constitue une légitimation " par le bas ». La seconde de ces modalités est le caractère non premier du métier d'écrivain, qui ne vient qu'après une expérience manuelle et parfois ingrate comme ici tuer des bêtes ou travailler à l'usine. Troisième modalité de légitimation : l'expérience des voyages dans cette présentation d'Antonin Varenne par le communiqué de presse de La Manufacture de Livres : "Antonin Varenne, alpiniste du bâtiment, charpentier, a travaillé en Islande, en Guyane, et aux États-Unis ${ }^{25}$ ». Les États-Unis demeurent ici un étalon de référence pour les auteur·trice's français·e·s de Country Noir. La quatrième modalité, c'est d'avoir vécu dans ces territoires ruraux reculés et retirés : les communicant·e·s éditoriaux·les et les journalistes culturel-le's ressassent à l'envi les origines rurales ou les lieux de vie campagnards des auteur·trice·s, l'argument de poids étant la longévité de ce séjour rural. C'est le cas pour Daniel Woodrell dans la communication de Rivages : «Daniel Woodrell est né dans le Missouri, dans la région des Ozarks, où il vit toujours ${ }^{26}$ ", en omettant bien sûr qu'il est allé vivre de nombreuses années en Louisiane. Enfin, la dernière façon $d^{\prime}$ '" en être ", c'est d'avoir connu la galère financière, la misère ou les addictions dans sa vie adulte. Laurence Biberfeld est présentée comme une exmarginale par la communication éditoriale : « Laurence Biberfeld a connu la ville et la rue. Rien dans les poches, le ventre vide. Plus tard, devenue institutrice, la vie l'amènera à quitter Paris... ${ }^{27}$ ».

Ces quelques exemples démontrent une recherche de l'authenticité et de la légitimité construite par l'interdiscours médiatique. Plus que la recherche d'une image "vraie ", c'est avant tout une certaine conception de la littérature qui est défendue à travers ces portraits et ces postures d'auteur.trice's au sein des communiqués de presse : une littérature réaliste, documentée et même vécue, proche du témoignage. Le Country Noir serait donc une littérature du bas, à l'écoute des voix oubliées, de l'infra-social et du marginal. L'authenticité construite par les communiqués de presse vise à rendre légitime la posture des auteur.trice's sur ces marges et régions reculées, loin d'une potentielle posture parisiano-centrée et dominante - dans le cas des auteur-trice-s français·e's.

On constate donc que la fortune médiatique de l'étiquette Country Noir se construit à la fois dans les discours amateurs aux États-Unis mais aussi dans le discours de la réception en France et, en particulier, du côté de la communication éditoriale. Il semble cependant que cette vague Country Noir ne perdura qu'un moment dans l'édition française : Aurélien Masson à la Série Noire a confié sa lassitude de cette catégorie de Country Noir, Cyril Herry a mis fin à sa carrière d'éditeur pour publier ses propres romans et Oliver Gallmeister a supprimé toutes les collections de sa maison d'édition pour s'orienter vers de la littérature générale étasunienne. Le Country Noir n'est-il donc qu'un effet de mode éditoriale et une construction médiatique? 


\section{Circulations transnationales et intermédiales du Country Noir}

Le Country Noir est en partie une construction médiatique : à partir d'une négociation dans l'interdiscours entre les différents acteur.trice's du champ culturel et d'une stabilisation progressive de l'expression, l'étiquette est utilisée médiatiquement par la stratégie de communication notamment des maisons d'édition françaises qui participent ainsi à sa légitimation en définissant un certain type de littérature et les auteur.trice's qui l'incarnent. Cette appellation aurait pu ne rester qu'une stratégie de communication ou un effet de mode mais c'était sans compter sur le succès intermédial du Country Noir, qui ne se cantonne pas qu'au champ éditorial et se définit sur d'autres supports visuels de la culture populaire comme la série télévisée et le cinéma. Nous essayons ici de démontrer que l'émergence progressive du Country Noir dans les imaginaires populaires - d'abord textuelle - se cristallise sur des supports visuels, permettant ainsi sa popularisation auprès du grand public. C'est en fait l'adaptation cinématographique en 2010 du dernier roman de la série de Monts Ozarks de Daniel Woodrell, Winter's Bone (2006), par la réalisatrice indépendante Debra Granik qui permet la diffusion de l'expression Country Noir et lui confère son identité visuelle. Le film de Granik a par ailleurs été adapté en bande dessinée en France dans la collection «Rivages/Casterman/Noir» par Romain Renard. On observe ainsi la plasticité et capacité de migration d'un support à l'autre de ce récit fondateur du Country Noir qu'est Winter's Bone, véritable œuvre prototypique du genre. Les fictions Country Noir s'articulent ainsi entre le romanesque, le cinématographique, le télévisuel et l'art graphique sur tous les supports de la culture médiatique : c'est que Jacques Migozzi appelle «les récits circulants ${ }^{28}$ ». Cette capacité de migration et cette circulation intermédiale fait la spécificité des fictions médiatiques dans un contexte technologique et culturel global. Il convient donc désormais de se demander, avec Jacques Migozzi : «Existerait-il dans ces conditions une esthétique propre à la culture médiatique, esthétique transformationnelle qui verrait les œuvres et les genres inscrire au cœur de leur projet poétique la virtualité de leurs mutations $?^{29}$ "

III. 4, 5 et 6 : Daniel Woodrell, Winter's Bone, New-York, Little, Brown \& Co, Hardcover, 2006. Debra Granik, Winter's Bone, Pretty Pictures, 2010. Romain Renard, Un hiver de glace, Rivages/Casterman/ Noir, 2011
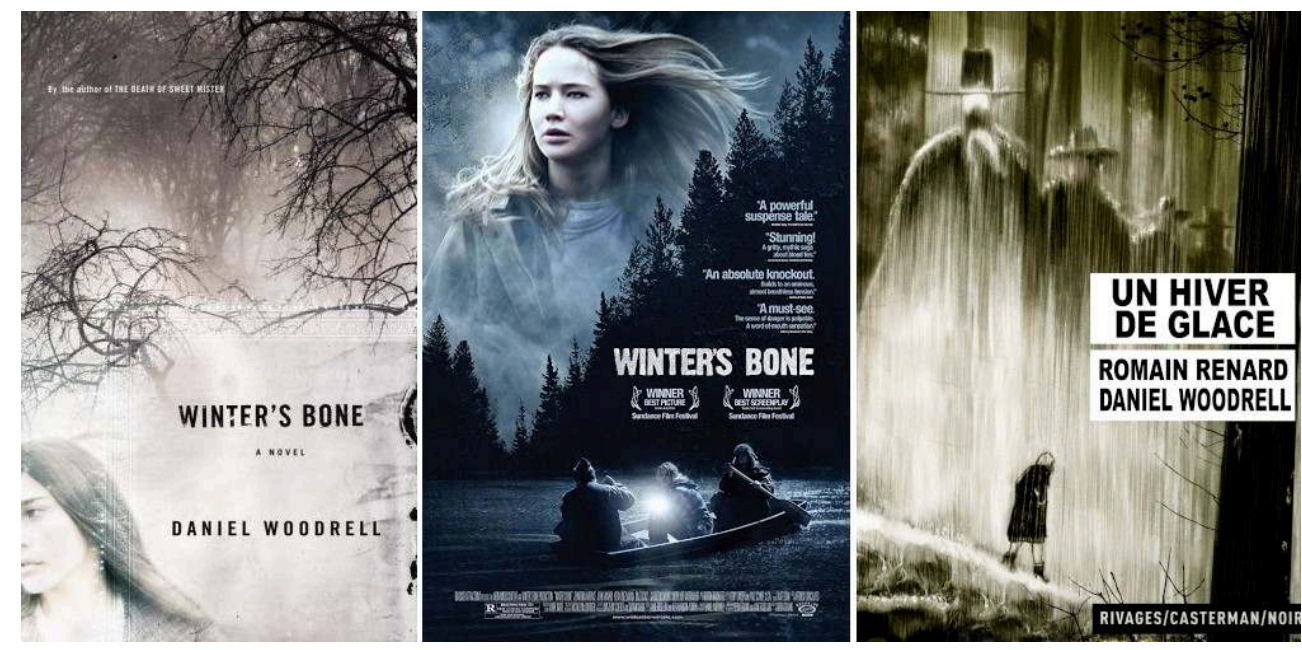
Winter's Bone, bien plus que Give Us a Kiss, constitue donc la fiction criminelle séminale du genre Country Noir. On trouve dans ce récit le personnel romanesque, constitué de " petit.e.s blanc.he.s pauvres ${ }^{30}$ » - hillbillies ou whitetrashes - vivant dans des régions rurales marginalisées et survivant de trafics et de prostitution, qui peuple la plupart des romans de Daniel Woodrell et autres récits de Country Noir. On trouve aussi cette grammaire visuelle typique de la plupart des fictions criminelles audiovisuelles relevant du Country Noir : une esthétique documentaire, mâtinée de tonalité gothique par l'inscription d'éléments ruinistes insérés dans le paysage, et rythmée par un tempo lent et contemplatif. Du côté des séries TV, celle qui a sans doute le plus marqué l'intrusion de l'esthétique Country Noir dans les imaginaires est la série True Detective (2014-), produite par HBO, dont la première saison se déroule dans les bayous de la Louisiane, la seconde dans le nord de la Californie, et la troisième dans les Monts Ozarks - encore eux. Cette série est d'autant plus emblématique que le rôle principal y est joué par l'acteur Matthew McConaughey qui devient véritablement l'incarnation physique et corporelle de l'anti-héros de Country Noir. Matthew McConaughey est un acteur texan né en 1969 qui, après avoir joué dans quelques comédies romantiques, s'est véritablement révélé dans des rôles de marginaux magnifiques dans des films Country Noir, notamment dans Killer Joe de William Friedkin (2011) et Mud de Jeff Nichols (2012). Son accent trainant du Sud, sa situation sociale aux limites de la marginalité et son éthique professionnelle le rapprochent de la figure du détective hardboiled.

La question se pose dès lors de savoir s'il est possible de transposer l'univers étasunien $\mathrm{du}$ Country Noir dans des productions visuelles en France. En d'autres termes : peut-il exister un Country Noir à la française ? En 2002, dans Western, France : la place de l'Ouest dans l'imaginaire français, Paul Bleton s'était posé la même question à propos du Western : sommes-nous du côté de la «distance culturelle " indépassable, selon les mots de Bleton? Ou au contraire, du côté de la «réappropriation»ou de la « reterritorialisation [...] made in France »? Ce qui est certain, c'est que l'engouement $\mathrm{du}$ public français pour les productions culturelles Country Noir va croissant et nous pouvons ainsi nous poser la même question que Bleton à propos du Western des années 1950 : «En quoi l'univers fictionnel du western permet-il de donner une forme à des préoccupations sociétales pertinentes en France, et très diverses ${ }^{31}$ ? ». Il nous semble que la réponse se trouve là encore en germe dans les pages de Bleton à propos du Western :

La situation incite à poser une question plus générale: quel genre la culture médiatique, à base urbaine, a-t-elle trouvé pour dire la dévitalisation, voire la désertification de la campagne, ce mouvement de fond de la société française durant le $\mathrm{xx}^{\mathrm{e}}$ siècle ? Visiblement pas le western, le retour antimoderne aux valeurs de la campagne ne pouvant évidemment pas se faire par l'Ouest, incarnation de la pop culture américaine [...] La campagne française, à l'aune des grands espaces sauvages, paraît bien médiocre, bien petite, bien policée, bien dépourvue d'aventure, peut-être trop informée par les valeurs de la littérature régionaliste ${ }^{32}$.

Paul Bleton dresse ici quatre constats. Le premier est que la culture médiatique doit prendre en charge le déclin des campagnes et la montée de l'urbanisation comme la grande métamorphose du $\mathrm{xx}^{\mathrm{e}}$ et du début du $\mathrm{xxI}^{\mathrm{e}}$ siècle. Ensuite, le Western - genre désormais calcifié parfois repris sur des modes référentiels ou parodiques - ne sera pas le genre de prédilection pour dire l'exode rural. Troisième constat: ce n'est pas d'un genre pop qu'émergera ce chant nostalgique de la fin du mode de vie campagnard. 
Dernier élément : la campagne française et les grands espaces étasuniens ne pourront jamais se retrouver dans un genre commun, comme le Nature Writing par exemple. Que reste-t-il alors ? Il reste le Country Noir. Un genre au fait des réalités rurales grâce à des auteur.trice's qui y ont vécu et en témoignent, un genre qui se démarque du Western et des grands espaces, un genre non pas pop mais bien populaire comme le sont les fictions criminelles, enfin un genre qui dresse des passerelles entre des situations sociales comparables, entre la France et les États-Unis. Cette situation sociale, c'est celles des populations blanches résidant dans des zones reculées et qui vivent avec rancœur cette marginalisation progressive. Le Country Noir est donc un genre populaire qui circule et gagne en légitimité dans un contexte globalisé car il dresse un constat politique dans lequel beaucoup de lecteur.trice's et spectateur.trice's se reconnaissent. Le rural constitue une nouvelle marginalité, ayant pris le relais des faubourgs des grandes villes du XIX ${ }^{e}$ siècle, et incarne ainsi une part oubliée et parfois terrifiante des modes de vie contemporains.

\section{Conclusion}

24 À travers des méthodes issues à la fois du close reading et du distant reading, nous avons donc démontré que l'étiquette Country Noir se construit progressivement et médiatiquement au sein d'une littérature populaire qu'est la littérature noire. Elle se stabilise d'abord dans l'interdiscours médiatique à travers des allers-retours entre le discours amateur étasunien et le discours éditorial français. Puis, elle se transforme en outil de vente dans les communiqués de presse des maisons d'édition au sein d'un régime médiatique qui dressent des postures et des trajectoires d'auteur-trice's de Country Noir relevant d'une recherche de l'authenticité et de l'image « vraie ». Au-delà d'une pure campagne marketing, ces communiqués de presse définissent en creux une certaine conception du Country Noir comme genre apte à parler du bas et par le bas. Enfin, le Country Noir se consolide de façon intermédiale par la circulation des supports artistiques initiée avec l'œuvre cinématographique séminale de Debra Granik, Winter's Bone en 2010 - adaptée d'un autre roman de Daniel Woodrell. C'est cette nouvelle grammaire visuelle du Country Noir qui permet notamment sa diffusion et sa popularisation au sein d'un large public, qui se reconnaît sans doute dans cette peinture de régions rurales en marge de la mondialisation. Le Country Noir est un genre qui, au sein d'un système globalisé et mondialisé, permet de redonner une existence aux marges géographiques et sociales. Plutôt qu'une instrumentalisation politique des voix oubliées - récupérées par l'extrême-droite aux États-Unis et dans toute l'Europe - le Country Noir permet de les prendre en charge dans leurs travers et leurs ambiguïtés sans jamais tomber dans le jugement mal venu. Nicolas Matthieu, citant Emil Cioran, définit ainsi le rôle de l'écrivaine de Country Noir : "On doit se ranger du côté des opprimés en toute circonstance, même quand ils ont tort, sans pourtant perdre de vue qu'ils sont pétris de la même boue que leurs oppresseurs ${ }^{33}$ ».

Rossi Marie-Laure, Écrire en régime médiatique : Marguerite Duras et Annie Ernaux : Actrices et spectatrices de la communication de masse, Paris, L'Harmattan, 2015. 


\section{Corpus primaire et interdiscours médiatique}

Communiqué de presse Rural Noir de Benoît Minville, Paris, Gallimard, « Série Noire » 2016.

Communiqué de presse Rural Noir de Benoît Minville, Paris, Gallimard, « Folio Policier », 2017.

Communiqué de presse Le Diable tout le temps de Donald Ray Pollock, Paris, Albin Michel, « Terres

d'Amérique », 2012.

Communiqué de presse Battues d'Antonin Varenne, Paris, La Manufacture de livres, "Territori », 2015.

Communiqué de presse Manuel du hors-la-loi de Daniel Woodrell, Paris, Payot\&Rivages, «Rivages Noir ", 2015.

Communiqué de presse Sous la neige, nos pas de Laurence Biberfeld, Paris, La Manufacture de livres, « Territori », 2017.

Granik Debra, Winter's Bone, Pretty Pictures, 2010.

Murphy Dwyer, « Daniel Woodrell : Southern Class » [en ligne], Guernica, 2013, disponible sur <https://www.guernicamag.com/southern-class/>, (consulté le 26 juillet 2019).

Schwenkstar, article « RURAL-COUNTRY THRILLERS » [en ligne], in IMDb, The Internet Movie Database, disponible sur <http://www.imdb.com/list/ls031295370/>, (consulté le 31 juillet 2019).

Woodrell Daniel, Faites-nous la bise (un roman noir rural), trad. Michèle Valencia, Rivages Noir, Paris, Payot \& Rivages, 1998.

\section{Corpus secondaire}

Bleton Paul, Western, France : la place de l'Ouest dans l'imaginaire français, Amiens, Encrage, 2002.

Eisenszweig Uri, Autopsies du roman policier, Paris, Union générale d'éditions, « 10/18 » 1983.

Jacquelin Alice, Genèse et circulations d'un paradigme culturel populaire en régime médiatique : le cas du Country Noir. France-États-Unis. 1996-2016, thèse de doctorat, Poitiers, 2019.

Laurent Sylvie, Thierry Leclère (éd.), De quelle couleur sont les Blancs ?: des « petits Blancs » des colonies au « racisme anti-Blancs », Paris, La Découverte, 2013.

Letourneux Matthieu, « Le genre comme pratique historique » [en ligne], Belphégor. Littérature populaire et culture médiatique (2016/14), disponible sur <http://journals.openedition.org/ belphegor/732>, (consulté le 26 juillet 2019).

Marion Philippe, « Narratologie médiatique et médiagénie des récits », Recherches en communication 7,1997 , pp. 61-88

Meizoz Jérôme, Postures littéraires : mises en scène modernes de l'auteur, Genève, Slatkine, 2007.

Meizoz Jérôme, L'Æil sociologique et la littérature, Genève, Slatkine, 2004. 
Migozzi Jacques, « Fictions transmédiatiques: du rhizome au réseau », in De l'écrit à l'écran. Littératures populaires : mutations génériques, mutations médiatiques. Actes du colloque international (12-15 mai 1998), Limoges, PULIM, 2000.

Moretti Franco, Distant reading, Londres, Verso, 2013.

\section{NOTES}

1. Cet article est en grande partie inspiré de ma thèse de doctorat sous la direction de Denis Mellier, Alice Jacquelin, Genèse et circulations d'un paradigme culturel populaire en régime médiatique: le cas du Country Noir. France-États-Unis. 1996-2016, Poitiers, 2019.

2. Daniel Woodrell, Faites-nous la bise (un roman noir rural), trad. Michèle Valencia, Paris, Payot \& Rivages, « Rivages Noir », 1998, p. 13.

3. Dwyer Murphy, «Daniel Woodrell : Southern Class» [en ligne], Guernica, 2013, disponible sur <https://www.guernicamag.com/southern-class/>, (consulté le 26 juillet 2019) : «Je veux bien que vous disiez de la Trilogie du Bayou qu'il s'agit de romans policiers. Je me suis intentionnellement glissé dans cette catégorie pour attirer certains lecteurs. J'aime beaucoup ce type de roman, et j'ai vraiment apprécié écrire ces livres. Mais ça me gênait beaucoup lorsque certaines personnes plaçaient Winter's Bone ou The Death of Sweet Mister dans la littérature de genre. On aurait dit que c'était juste ma réputation qui me poursuivait. L'expression "Country Noir" ne mérite plus d'être utilisée. Le noir a été rebattu à mort ces derniers temps, il y en a tellement de types maintenant. Et puis, je ne veux pas être obligé de coller à ma propre définition. " Je traduis.

4. Matthieu Letourneux, «Le genre comme pratique historique » [en ligne], Belphégor. Littérature populaire et culture médiatique (2016/14), disponible sur <http://journals.openedition.org/ belphegor/732>, (consulté le 26 juillet 2019).

5. Matthieu Letourneux, Ibid : «Pourquoi prétendre que le roman policier, la science-fiction, la chick lit ou l'anticipation gagnent à être pensés à travers une définition transcendante s'ils sont de fait le produit d'un interdiscours situé dans le temps et l'espace ? Pourquoi repousser les usages, si dans le fond ce sont ces usages qui ont imposé, au fil du temps et de la variation sérialisée des textes, un concept qui auparavant n'existait pas indépendamment d'eux?».

6. Philippe Marion, « Narratologie médiatique et médiagénie des récits », Recherches en communication 7, 1997, pp. 61-88.

7. Jérôme Meizoz, Postures littéraires : mises en scène modernes de l'auteur, Genève, Slatkine, 2007.

8. Pour un référencement précis des sites et des archives utilisés, voir la bibliographie in Alice Jacquelin, Genèse et circulations d'un paradigme culturel populaire en régime médiatique : le cas du Country Noir. France-États-Unis. 1996-2016, Poitiers, pp. 446-447 et pp. 461-476.

9. Schwenkstar, article «RURAL-COUNTRY THRILLERS» [en ligne], in IMDb, The Internet Movie Database, disponible sur <http://www.imdb.com/list/ls031295370/>, (consulté le 31 juillet 2019). Nous traduisons en conservant les concepts culturels étasuniens en italiques.

10. Uri Eisenszweig (éd.), Autopsies du roman policier, Paris, Union générale d'éditions, "10/18 », 1983, p. 8.

11. Matthieu Letourneux, «Le genre comme pratique historique » [en ligne], Belphégor. Littérature populaire et culture médiatique. 14 | 2016, consulté le 29 mars 2021. URL : http:// journals.openedition.org/belphegor/732

12. Ibid.

13. Cette institution patrimoniale est l'un des lieux stratégiques de la recherche en culture médiatique et littératures populaires. La fonte des dotations étatiques allouées ainsi que la réduction drastique des postes et surtout la perte du dépôt légal qui lui était jusqu'ici dédié 
constitue une menace pour la pérennité de ses missions de documentation du grand public, de rencontre des chercheurs, d'archivage des fonds patrimoniaux extrêmement précieux et de médiation culturelle.

14. Pour une explication extensive de la méthodologie scientifique et des techniques utilisées, se reporter au chapitre 2 de Alice Jacquelin, Genèse et circulations d'un paradigme culturel populaire en régime médiatique: le cas du Country Noir. France-États-Unis. 1996-2016, Poitiers, pp. 120-122.

15. Voir à ce sujet, les définitions et les propositions de Franco Moretti in Distant reading, Londres, Verso, 2013. Il nous semble néanmoins que ces deux postures sont tout à fait compatibles et s'enrichissent l'une l'autre. Le distant reading tel que défini par Moretti s'articule en effet à des analyses littéraires plus précises pour l'analyse de la représentation des marges et des campagnes dans le polar.

16. Communiqué de presse Rural Noir de Benoît Minville, Paris, Gallimard, « Série Noire », 2016.

17. Communiqué de presse Rural Noir de Benoît Minville, Paris, Gallimard, «Folio Policier », 2017.

18. Sur ce rôle des éditeur.trice's comme passeur'euse's de textes et de genres littéraires étrangers, voir le chapitre 3 "Sociabilités, acteurtrice's et réseaux : la constitution du Country Noir comme champ littéraire " in Alice Jacquelin, Genèse et circulations d'un paradigme culturel populaire en régime médiatique: le cas du Country Noir. France-États-Unis. 1996-2016, Poitiers, pp. 182-190.

19. Pour une étude de cette «territorialisation» du polar limousin, voir Alice Jacquelin, "Territorialisation du polar européen, entre représentation pittoresque et écriture des marges », Belphégor, à paraître; Natacha Levet, " Noirs desseins en Limousin », in Thomas Bauer (dir.), L'écrivain et son Limousin, Limoges, PULIM, 2013, pp. 107-120.

20. "Entretien avec Cyril Herry » in Alice Jacquelin, Genèse et circulations d'un paradigme culturel populaire en régime médiatique: le cas du Country Noir. France-États-Unis. 1996-2016, Poitiers, pp. 13-17. 21. Marie-Laure Rossi, Écrire en régime médiatique: Marguerite Duras et Annie Ernaux: Actrices et spectatrices de la communication de masse, Paris, L'Harmattan, 2015, pp. 12-13.

22. Jérôme Meizoz, Postures littéraires : mises en scène modernes de l'auteur, Slatkine, Genève, 2007.

23. Jérôme Meizoz, L'œil sociologique et la littérature, Slatkine, Genève, 2004, p. 51.

24. Communiqué de presse Le diable tout le temps de Donald Ray Pollock, Paris, Albin Michel, 2012.

25. Communiqué de presse Battues d'Antonin Varenne, Paris, La Manufacture de livres, « Territori », 2015.

26. Communication Manuel du hors-la-loi de Daniel Woodrell, Paris, Payot \& Rivages, « Rivages/ Noir ", 2015.

27. Communication de presse Sous la neige, nos pas de Laurence Biberfeld, Paris, La Manufacture de livres, « Territori », 2017.

28. Jacques Migozzi, «Fictions transmédiatiques: du rhizome au réseau », in De l'écrit à l'écran. Littératures populaires: mutations génériques, mutations médiatiques. Actes du colloque international (12-15 mai 1998), Limoges, PULIM, 2000, p. 10.

29. Ibid., p. 17.

30. Sur le problème de la traduction de l'expression étasunienne whitetrash par celle « petit blanc pauvre " issue de la colonisation française, voir l'ouvrage de Sylvie Laurent et Thierry Leclere (éd.), De quelle couleur sont les Blancs?: des "petits Blancs" des colonies au "racisme anti-Blancs", Paris, La Découverte, 2013.

31. Paul Bleton, Western, France : la place de l'Ouest dans l'imaginaire français, Amiens, Encrage, 2002, p. 136.

32. Ibid., p. 222.

33. "Entretien avec Nicolas Mathieu " in Alice Jacquelin, Genèse et circulations d'un paradigme culturel populaire en régime médiatique: le cas du Country Noir. France-États-Unis. 1996-2016, Vol. II, Poitiers, pp. 5-12. 


\section{RÉSUMÉS}

Si l'expression « Country Noir » a été inventée en 1996 par l'écrivain étasunien de romans noirs, Daniel Woodrell, pour sous-titrer son roman Give Us a Kiss, a Country Noir, l'étiquette ne devient prolifique que dans les années 2010. Le Country Noir, qui relève de la fiction criminelle située en milieu rural, est un genre qui se construit médiatiquement selon trois modalités. L'expression est d'abord reprise par l'interdiscours médiatique dans une forme de négociation discursive entre les différents acteurs du champ culturel. Elle est ensuite transformée en étiquette dans les stratégies de communication des maisons d'édition françaises qui participent ainsi de sa légitimation. Enfin, elle se consolide dans la circulation transnationale, entre la France et les États-Unis, d'œuvres intermédiales sur des supports textuels et visuels tels que le roman policier, le cinéma et les séries télévisées. Cet article a pour objectif de démontrer la dynamique et les mouvances d'un genre fictionnel populaire en émergence.

\section{INDEX}

Mots-clés : country noir, roman noir, fiction criminelle, culture médiatique, ruralité

\section{AUTEUR}

\section{ALICE JACQUELIN}

Alice Jacquelin est docteur en littérature générale et comparée, spécialiste du roman noir contemporain. Elle est postdoctorante à l'Université de Limoges (EHIC, EA 1087) au sein du projet européen DETECt sur les fictions criminelles, pour lequel elle a été cocommissaire de l'exposition L'Europe du polar (BiLiPo 2020). Elle fait partie du bureau de l'association scientifique LPCM.

Publications :

Jacquelin Alice, «Identity, Borders and the Environment: New Political Issues in Contemporary French Noir ", Academic Quarter/Akademisk Kvarter, vol. 22, Spring 2021, pp. 112-23. https://doi.org/10.5278/ojs.academicquarter.vi22.6605 Jacquelin Alice, «L'indice policier, une trace indécidable? », in M. Deperne, S. DichyMalherme, L. Pichard (dirs.), Trace(s), Limoges, PULIM, 2018, pp. 229-241.

alice.jacquelin@unilim.fr 